\title{
The prognostic significance of fibroblast activation protein- $\alpha$ in human lung adenocarcinoma
}

\author{
Jianlin Shi ${ }^{1,2 \#}$, Zongliu Hou ${ }^{2,3 \#}$, Jun Yan ${ }^{2,3}$, Wanfang Qiu ${ }^{3,4}$, Luxin Liang ${ }^{2,3}$, Mingyao Meng ${ }^{2,3}$, Lin Li $^{2,3}$, \\ Xiaodan Wang ${ }^{2,3}$, Yanhua Xie ${ }^{2,3}$, Lihong Jiang ${ }^{2,5}$, Wenju Wang ${ }^{2,3}$ \\ ${ }^{1}$ Department of Thoracic Surgery, Yan'an Affiliated Hospital of Kunming Medical University, Kunming 650051, China; ${ }^{2}$ Key Laboratory of Yan'an \\ Affiliated Hospital of Kunming Medical University, Kunming 650051, Yunnan, China; ${ }^{3}$ Key Laboratory of Tumor Immunological Prevention and \\ Treatment of Yunnan Province, Kunming 650051, China; ${ }^{4}$ Department of Ultrasound, Kunming Children's Hospital, Kunming 650228, China; \\ ${ }^{5}$ Department of Cardiovascular Surgery, The First People's Hospital of Yunnan Province, Kunming 650051, China \\ Contributions: (I) Conception and design: L Jiang, W Wang, Z Hou; (II) Administrative support: L Jiang, Z Hou, W Wang; (III) Provision of study \\ materials or patients: J Shi, J Yan; (IV) Collection and assembly of data: J Shi, W Qiu, L Liang; (V) Data analysis and interpretation: J Shi, W Qiu, L \\ Liang; (VI) Manuscript writing: All authors; (VII) Final approval of manuscript: All authors. \\ \#These authors contributed equally to this work \\ Correspondence to: Lihong Jiang; Wenju Wang. Yan'an Affiliated Hospital of Kunming Medical University, No. 245 East of Renmin Road, Kunming \\ 650051, China. Email: jianglihong_yayy@163.com; wangwenju_vip@hotmail.com
}

Background: Fibroblast activation protein (FAP) is a type II cell surface-bound integral serine protease, which is an important biomarker of cancer-associated fibroblasts. FAP- $\alpha$ performs several biological activities, including remolding extracellular matrix and acting as an immunosuppressor in the tumor microenvironment. However, the proliferation role of FAP- $\alpha$ in human lung adenocarcinoma has not been fully elucidated.

Methods: The expression of FAP- $\alpha$ in 94-paired human lung adenocarcinoma tissues was identified by immunohistochemistry test. The effect of FAP on cell proliferation was examined by CCK-8 assay. RNAsequencing and bioinformatics analysis were utilized to investigate the underlying mechanism. Western blot analysis, quantitative polymerase chain reaction (qPCR), and nude mice experiments, were also conducted for further validation.

Results: The proliferation rates of human fibroblast strains FAP-HFF and FAP-BJ, and human lung adenocarcinoma cell line FAP-SPC-A-1 were higher than those of controls. The nude mice experiment also showed that FAP could promote the proliferation of SPC-A-1 cell line in vivo. qPCR and Western blot analysis indicated that CCNB1 was upregulated by the overexpression of FAP in the lung adenocarcinoma cell line. The expression of FAP- $\alpha$ was higher in both the cytoplasm and stroma of lung adenocarcinoma than in adjacent normal tissues. Survival analysis indicated that patients with higher expression of FAP- $\alpha$ in tumor stroma had a poor prognosis $(\mathrm{P}=0.019)$. The Cancer Genome Atlas Program (TCGA) data also showed that the expression of FAP within tumor tissues was higher (in both cytoplasm and stroma) compared with that in normal tissues $(\mathrm{P}<0.05)$.

Conclusions: Our study indicates that FAP- $\alpha$ could facilitate the proliferation of lung adenocarcinoma cells and can be a prognostic marker in human lung adenocarcinoma.

Keywords: Fibroblast activation protein (FAP); lung adenocarcinoma; prognosis; proliferation

Submitted Dec 04, 2019. Accepted for publication Dec 24, 2019.

doi: 10.21037/atm.2020.01.82

View this article at: http://dx.doi.org/10.21037/atm.2020.01.82 


\section{Introduction}

Due to an aging population, the deterioration of the earth's environment, smoking habits, genetic background, and other factors, lung cancer remains the leading cause of cancer-related deaths worldwide (1). A growing body of research suggests that the initiation and progression of tumors are the results of a dynamic interaction between tumor cells and various components within the tumor microenvironment (TME), which can include immune cells, endothelial cells, and fibroblasts (2-6). The tumor microenvironment may therefore be a promising target for cancer therapy (7).

Human fibroblast activation protein (FAP) is a highly significant type II serine protease and has a molecular weight of $95 \mathrm{kDa}$. It is composed of two subunits, with one of these subunits, the $\alpha$-subunit, being the primary determinant of serine protease activity (8). FAP is mainly expressed on the surface of activated fibroblasts in $90 \%$ of epithelial-derived cancers, transiently expressed in mesenchymal cells of bone marrow, wound healing tissues, and some fibrosis tissues, but not expressed in benign lesions (9). By virtue of its specific expressional patterns, FAP is widely considered an ideal biomarker of TME (10). Recent research indicates that the high expression of FAP in some tumors can often predict poor prognosis in malignancies like colorectal cancer (11), esophageal squamous cancer (12), and lung squamous cell carcinoma (13). However, FAP may also play a restraining role in other tumors (14-17).

\section{Methods}

\section{Cell lines and culture}

The human fibroblast cell lines HFF and BJ, and human lung adenocarcinoma cell line SPC-A-1 used in this study were obtained from the Cell Library of Typical Culture Preservation Committee of the Chinese Academy of Sciences in 2010 . All were routinely cultured at $37^{\circ} \mathrm{C}$ in a humidified air atmosphere containing $5 \%$ carbon dioxide in Dulbecco's Modified Eagle Medium (DMEM) or RPMI 1640 supplemented with $10 \%$ fetal bovine serum (FBS) (BI), $100 \mathrm{mg} / \mathrm{mL}$ penicillin (Sigma-Aldrich), and $100 \mathrm{mg} / \mathrm{mL}$ streptomycin (Sigma-Aldrich). All cell lines were used within 20 passages and thawed fresh every 2 months. These cell lines were free of Mycoplasma contamination and authenticated by quality examinations of morphology and growth profile.

\section{Human adenocarcinoma samples}

One cohort of tissue microarrays was purchased from Shanghai Outdo Biotech Co., Ltd., China, which contained 94-paired human lung adenocarcinoma samples and their matched adjacent noncancerous tissues (clinical data shown in Table S1). The matched adjacent noncancerous tissues were taken at a distance of at least $3 \mathrm{~cm}$ from the tumor. All human specimens were approved by the Ethical Review Committee of the World Health Organization of the Collaborating Center for Research in Human Production authorized by the Shanghai Municipal Government and obtained with informed consent from all patients. This study was approved by the Ethics Committee of Kunming Medical University.

\section{Immunobistochemistry (IHC) staining}

Paraffin blocks were sectioned at a thickness of $4 \mathrm{~mm}$. The wax was melted at $63{ }^{\circ} \mathrm{C}$ overnight. The sections were deparaffinized in xylene, rehydrated in graded alcohol series, and boiled in $0.01 \mathrm{M}$ citrate buffer ( $\mathrm{pH}$ 6.0) for $2 \mathrm{~min}$ in an autoclave. Endogenous peroxidase activity was blocked using hydrogen peroxide $(0.3 \%)$, followed by incubation with $5 \%$ bovine serum albumin to reduce nonspecific binding. Tissue sections were incubated with an anti-FAP sheep polyclonal antibody (1:50 dilution) (R\&D System). The slides were rinsed with phosphatebuffered saline (PBS), and a secondary antibody (sheep) was applied at a 1:500 dilution in PBS for $30 \mathrm{~min}$ at room temperature. After rinsing with PBS for $30 \mathrm{~s}$, the slides were incubated with streptavidin-HRP for $30 \mathrm{~min}$ at room temperature, rinsed with PBS, incubated for $15 \mathrm{~min}$ with the chromogen 3,3-diaminobenzidine, and counterstained with hematoxylin. The slides were then examined under a transmission light microscope. The cytoplasmic and stromal staining of FAP in tumor tissue was scored by a semi-quantitative method reported previously (18). The percentage of positive cells was scored as follows: grade 0 (absent or $<1 \%$ ), grade $1(1-25 \%)$, grade $2(26-50 \%)$, grade $3(51-75 \%)$, and grade 4 (76-100\%). The staining intensity was also graded $(0,1+, 2+$, and $3+)$. The immunoreactivity score (IS) was determined by multiplying the intensity score and percentages of positive cells. The IS ranged from 0 to 12. The cutoff threshold for this scoring system was set as 3 , and a score of $>3$ was considered as high FAP expression and $\leq 3$ as low FAP expression according to the previous study (13). 


\section{Transfection of target cells with FAP-overexpressing plasmids with lentivirus}

Using the FAP gene as the template, the overexpressing and negative lentiviral vectors were purchased from Shanghai Genechem Co., Ltd., China. HFF, BJ, and SPC-A-1 cells were seeded in six-well plates at a concentration of $1.0 \times 10^{5}$ cells per well $(15-30 \%$ confluence) on the day before transduction. FAP-overexpressing lentivirus [LVFAP (8709-1)] was transduced into cells at a multiplicity of infection of 100 (SPC-A-1) or 20 (HFF, BJ) using polybrene $(5 \mu \mathrm{g} / \mathrm{mL})$ and Enhanced Infection Solution (Genechem, China). At the same time, a negative control virus CON145 (Genechem) was transduced into cells using the same methods to control the impact of the viral vector.

\section{Cell proliferation assay (cell counting kit-8)}

Target cells were collected and seeded into 96-well plates in the right concentration $\left(1 \times 10^{3}-2 \times 10^{3} /\right.$ well $)$. The seeded plates were incubated in a humidified chamber $\left(37^{\circ} \mathrm{C}\right.$, $5 \% \mathrm{CO}_{2}$ ) for $24 \mathrm{~h}$ (cell line dependent) to permit cell attachment. The next day, $10 \mu \mathrm{L}$ of cell counting kit- 8 (CCK-8) solution was added to each hole, and the culture plate was incubated for 1-4 h. The absorbance at $450 \mathrm{~nm}$ was detected using an enzyme-labeling instrument. The experiments were repeated independently 3 times.

\section{RNA-sequencing analysis}

Total RNA from cells (FAP-HFF, FAP-BJ, and its controls) was extracted using TRIzol reagent (Invitrogen, CA, USA). The mRNA samples were given to Novel Bioinformatics Company (Shanghai, China) for transcriptional sequencing analysis. Genes differentially expressed between the experimental and control groups were annotated using the Kyoto Encyclopedia of Genes and Genomes database. The significance of all pathways was calculated using a hypergeometric distribution-based Fisher's exact test. Multiple hypothesis testing results were calibrated to obtain the false discovery rate. Significantly overrepresented pathways among the differentially expressed genes were selected using $<0.05$ as the cutoff for statistical significance.

\section{Real-time quantitative polymerase chain reaction}

Total RNA from cells was isolated using TRIzol as recommended by the manufacturer (Promega Company).
The concentration and purity of the total RNA were assessed using an ultraviolet-visible spectrophotometer (NanoDrop2000/2000c, Thermo). Total RNA was reversetranscribed using the Promega M-MLV Reagent kit (Promega, China). Quantitative polymerase chain reaction (qPCR) was performed with the CFX96 Real-Time System (Bio-Rad, USA) using SYBR Master Mixture (Bio-Rad). $\beta$-actin was used as an internal control. The relative expression of mRNAs was calculated using the $2^{\Delta \mathrm{Ct}}$ method, where $\Delta \mathrm{Ct}=\mathrm{Ct}$ of the objective gene - Ct of $\beta$-actin, and then transformed to $\log 2$. Each reaction was repeated at least 3 times independently.

\section{Western blotting}

Protein extracts from cells were prepared in RIPA buffer (10 mM Tris, $\mathrm{pH} 7.5,0.1 \mathrm{M} \mathrm{NaCl}, 1 \mathrm{mM}$ EDTA, and 0.1\% Triton $\mathrm{X}-100)$. The protein was subjected to $12 \%$ sodium dodecyl sulfate-polyacrylamide gel electrophoresis and electrotransferred to nitrocellulose membranes (Millipore). The membranes were blocked with TBST buffer $(20 \mathrm{mM}$ Tris, $\mathrm{pH} 7.5,500 \mathrm{mM} \mathrm{NaCl}, 0.05 \%$ Tween-20, and $0.2 \%$ Triton X-100) containing 5\% skimmed milk and then incubated with the primary antibodies against different proteins at $4{ }^{\circ} \mathrm{C}$ overnight, followed by incubation with a secondary antibody. The bands were visualized using the ECL Plus Western blotting system (Amersham). The antibodies used in Western blot analysis included antiFAP (AF3715, RD), anti- $\beta$-actin (Abcam) and anti-CCNB1 (Proteintech).

\section{Protocols and animals}

Four-week-old female BALB/c nude mice were obtained from Hunan Silaikejingda Company. These mice were maintained in a specific pathogen-free unit under isothermal conditions. All the experimental procedures were conducted in accordance with the Institutional Animal Committee of Kunming Medical University. FAP-SPC-A-1 and control cells $\left(2.0 \times 10^{7} / \mathrm{mL}\right)$ were suspended in $100 \mu \mathrm{L}$ of PBS. The cells were then subcutaneously injected into the back of nude mice to form xenograft tumors. After about 21 days, all mice were confirmed dead after a complete coma and a dislocation of the cervical spine. Medical scissors and medical tweezers were used to dissect the mice and observe their lungs and livers to detect metastasis.

\section{The Cancer Genome Atlas data analysis}

Gene expression data of 546 samples (488 tumors and 58 
matched noncancerous tissues) from lung adenocarcinoma types and clinical data were downloaded from The Cancer Genome Atlas (TCGA, http://cancergenome.nih.gov, RNA-Seq Version 2). Statistical analyses were performed using the R software (V 3.0.3) (http://www.R-project. org/). $\mathrm{P}$ values of less than 0.05 were considered statistically significant.

\section{Statistical analysis}

All data were displayed as the mean \pm standard deviation and processed using SPSS 17.0 statistical software. The differences in the expression of FAP between tumor and adjacent tissues were tested using paired-sample $t$-test analysis. The correlations among clinical data were analyzed by Spearman and Pearson methods. Survival analysis was performed using Kaplan-Meier and log-rank methods. A $\mathrm{P}$ value of less than 0.05 was considered statistically significant.

\section{Results}

Effects of FAP- $\alpha$ on the proliferation of lung

adenocarcinoma cell in vitro and in vivo

In order to investigate the effect of FAP- $\alpha$ on the proliferation of lung adenocarcinoma and stroma cells, HFF, BJ, and SPC-A-1 cells were transfected with FAP- $\alpha-$ overexpressing and negative control lentivirus. After three days of transfection, the green fluorescent protein (GFP) expression was observed under a fluorescence microscope. The transfection efficiencies were more than $80 \%$, and the cell status was normal (Figure S1). The protein levels of FAP- $\alpha$ were detected by Western blot among these cell lines (Figure $1 A, B$ ). The CCK-8 assay was applied to detect the proliferation ability of various cell strains after the overexpression of FAP- $\alpha$. The results showed that FAP$\alpha$-overexpressing cells had a higher proliferation ability compared with negative controls $(\mathrm{P}<0.05)$ (Figure $1 C$ ). Next, we confirmed the in vivo results using human adenocarcinoma xenografts in nude mice. The nude mice were inoculated with FAP-SPC-A-1 cells and control cells. The results also showed that FAP- $\alpha$ could facilitate the proliferation of lung adenocarcinoma in vivo (Figure 1D,E).

\section{Gene expression profile and gene ontology analysis}

By comparing the differential gene expressions of HFF and
BJ cell lines between FAP over-expression groups (FAP$\mathrm{HFF}, \mathrm{FAP}-\mathrm{BJ}$ ) and negative control groups (NC-HFF, $\mathrm{NC}-\mathrm{BJ})$, it was found that there were 1,172 genes in the HFF groups and 304 genes in BJ groups (Figure 2A). Gene ontology analysis indicated that FAP- $\alpha$ might be involved in multiple biological processes, including angiogenesis, extracellular matrix organization, and mitotic nuclear division (Figure 2B,C). Pathway analysis further indicated that pantothenate and coenzyme A biosynthesis, focal adhesion, and other signaling pathways might be activated by overexpressed FAP- $\alpha$ (Figure $2 D, E$ ).

\section{Effects of FAP- $\alpha$ on cell cycle, angiogenesis, and cell proliferation patbways}

Subsequently, qPCR was used to verify the CCNB1, CCNB2, MMP3, ITGB1, EFEMP1, and AKT in HFF cells, and the CCNB1, CCNB2, EGF, TGFA, AKT, PIK3R3, ITGB1, and EFEMP1 in SPC-A-1 cells. The results showed that MMP3 was less expressed in FAP-HFF cells than in NC-HFF cells $(\mathrm{P}<0.05$, Figure $3 A)$. EGF and TGFA were more downregulated in the FAP-SPC-A-1 cells than in the NC-SPC-A-1 cells, while CCNB1 and CCNB2 were more expressed in the FAP-SPC-A-1 cells than in the NCSPC-A-1 cells $(\mathrm{P}<0.05$, Figure $3 B)$. Western blot analysis also confirmed that FAP- $\alpha$ could upregulated the CCNB1 expression more in the FAP-SPC-A-1 cells than in the NCSPC-A-1 cells (Figure 3C).

\section{FAP- $\alpha$ expression in buman lung adenocarcinoma}

After removal of those cases without clinical stage and or that were lost during follow-up, 75 cases remained in the survival analysis with 5-10 years of follow-up. Clinical characteristics of all patients are listed in Table S1. FAP- $\alpha$ expression was detected both in the cytoplasm of tumor cells and stromal cells, while the positive rates were quite different in these cells (Figure 4A). Out of the 92 lung adenocarcinoma cases studied, 29 were considered as high FAP- $\alpha$ expression in tumor cytoplasm (31.52\%), and 63 were defined as low FAP- $\alpha$ expression in tumor cytoplasm $(68.48 \%)$, while the expression of FAP- $\alpha$ in the cytoplasm of adjacent tissues was also low (83 cases). Our studies also found that 49 cases were considered as high FAP- $\alpha$ expression in tumor stroma (53.26\%), and 43 cases were defined as low FAP- $\alpha$ expression in tumor stroma (46.74\%), while almost all cases were considered as low FAP- $\alpha$ expression in the stroma of adjacent tissues. A paired non- 
A
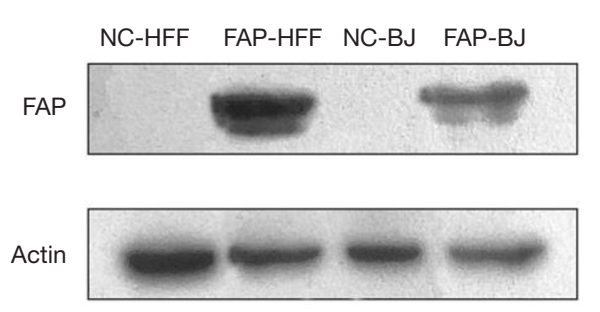

B

NC-SPC-A-1 FAP-SPC-A-1
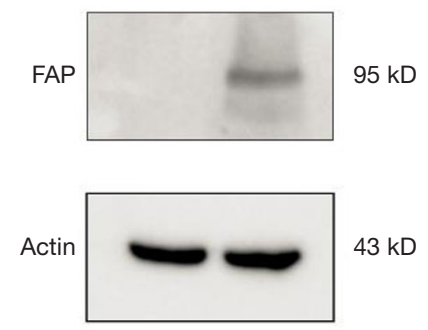

C
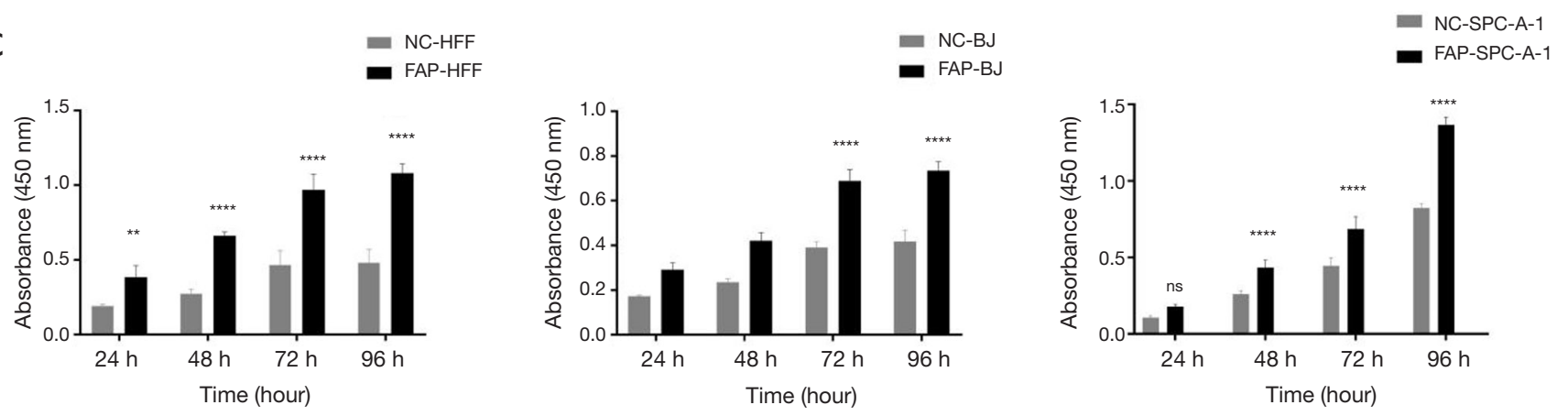

D
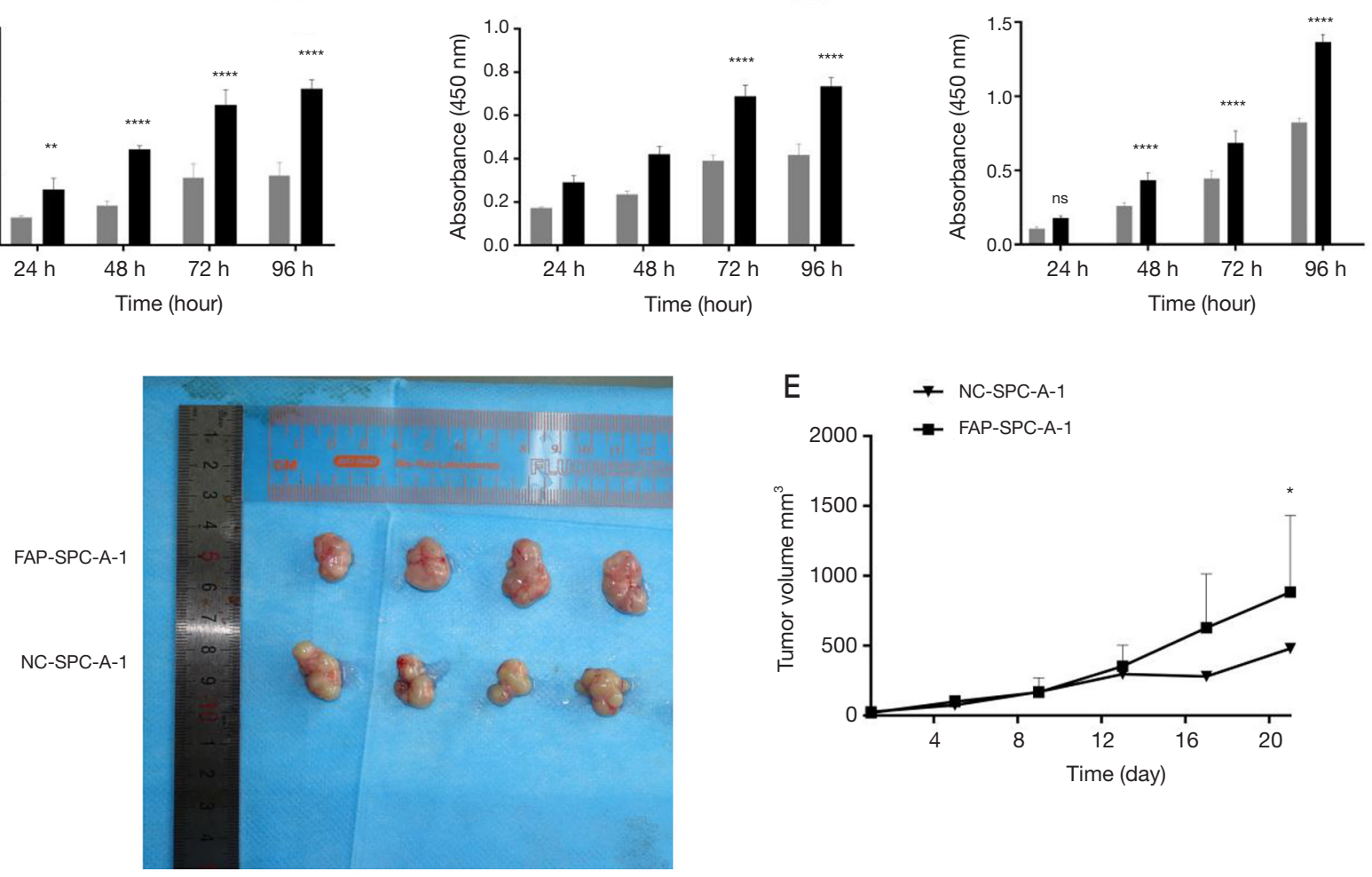

Figure 1 Effect of FAP- $\alpha$ on the proliferation of lung adenocarcinoma cells in vitro and in vivo. (A,B) The expressions of FAP in HFF, BJ, and SPC-A-1 cell lines after transfection with FAP-overexpressing and control plasmids were verified by Western blot analysis; (C) FAPoverexpressing cells had a higher proliferation ability compared with control cells $(\mathrm{P}<0.05)$; (D,E) after being inoculated with FAP-SPC-A-1 cells and its control cells, the tumor volumes of nude mice with FAP-SPC-A-1 cells were higher than with NC-SPC-A-1 cells $(\mathrm{P}<0.05)$. *, $\mathrm{P}<0.05 ;{ }^{* *}, \mathrm{P}<0.01 ;{ }^{* * * *}, \mathrm{P}<0.0001$.

parametric pairing Wilcoxon and Chi-Square inspection test revealed that FAP- $\alpha$ expression was significantly higher in both tumor cytoplasm and stroma than in adjacent tissues $(\mathrm{P}<0.05$; Table 1). The clinicopathological analysis revealed that FAP- $\alpha$ expression was only negatively correlated with the age of patients (Tables S2,S3) $(\mathrm{P}<0.05)$.

\section{Survival analysis of FAP- $\alpha$ expression in lung adenocarcinoma patients}

The survival analysis of FAP- $\alpha$ expression in human adenocarcinoma patients was determined by Kaplan-Meier and log-rank test. When detecting the staining of FAP- $\alpha$ 
A

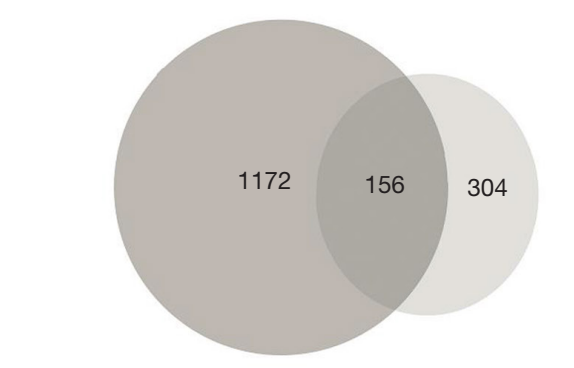

FAP-HFF vs. NC-HFF FAP-BJ vs. NC-BJ
B
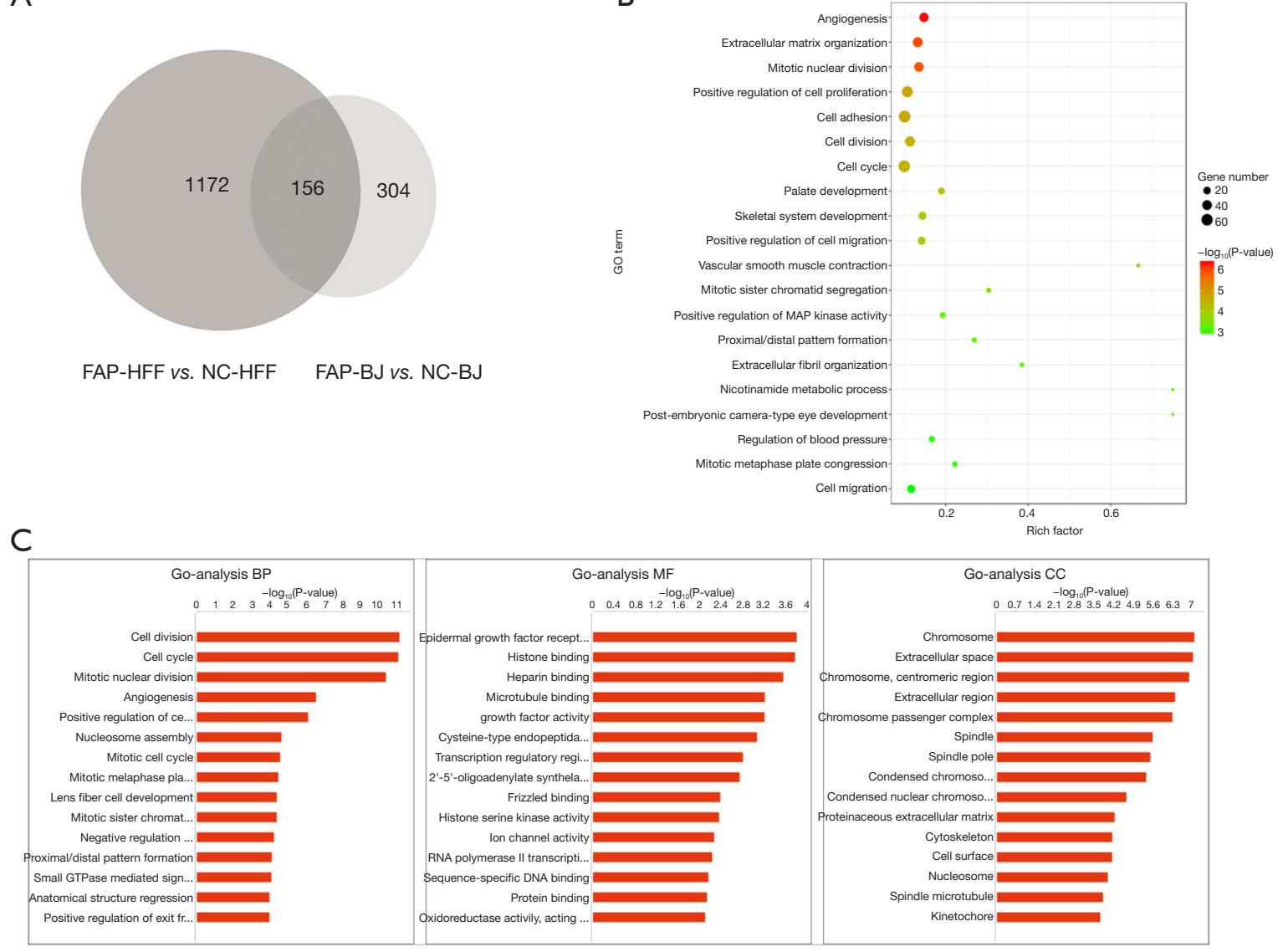

D

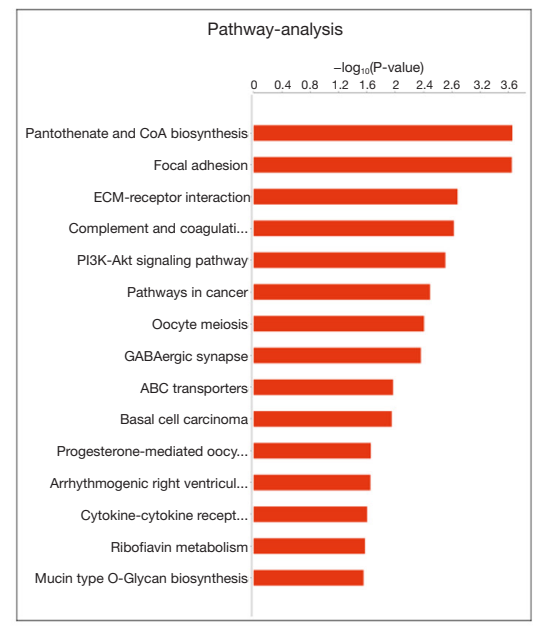

E

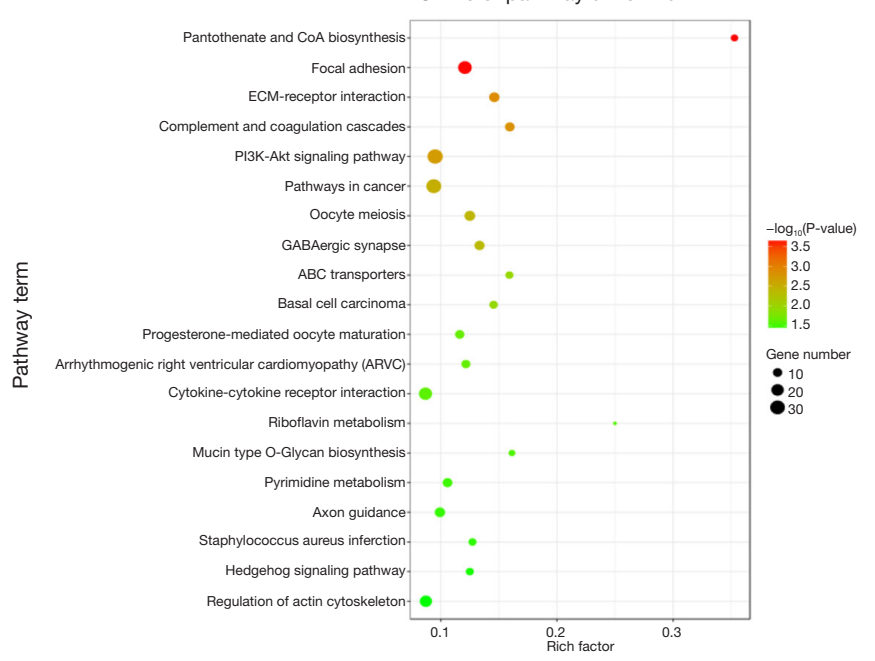

Figure 2 Gene expression profile and gene ontology analysis. (A) By comparing the differential gene expressions of HFF and BJ cell lines in FAP over-expression groups (FAP-HFF, FAP-BJ) and negative control groups (NC-HFF, NC-BJ), it was found that there were 1,172 genes in the HFF groups and 304 genes in the BJ groups. (B,C) Gene ontology analysis indicated that FAP- $\alpha$ might be involved in multiple biological processes, including angiogenesis, extracellular matrix organization, and mitotic nuclear division. (D,E) Pathway analysis further indicated that pantothenate and CoA biosynthesis, focal adhesion, and another pathways may be activated by FAP- $\alpha$. BP, biological process; MF, molecular function; CC, cellular component. 
A

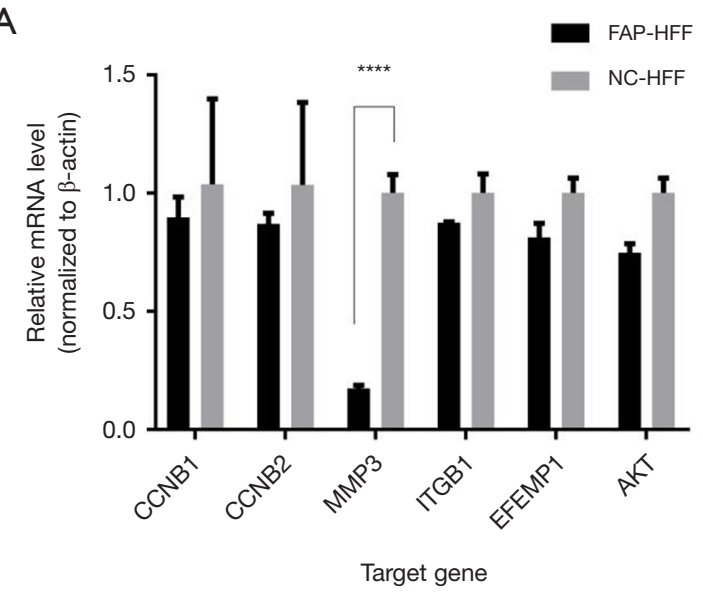

B

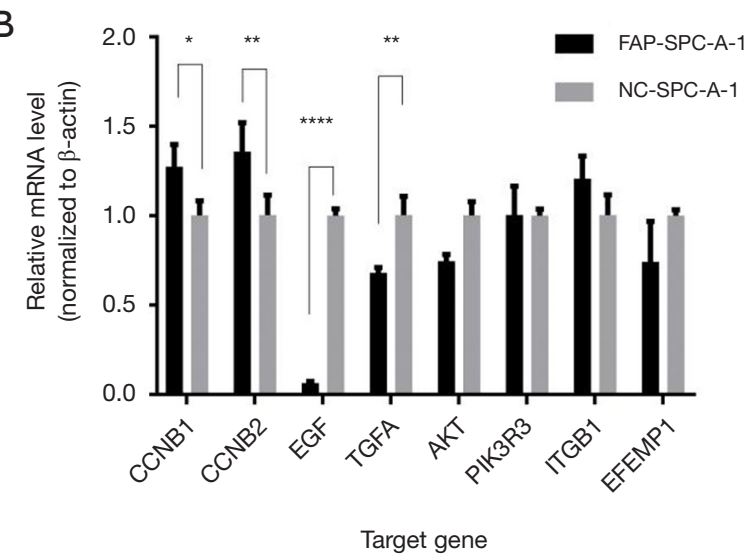

C

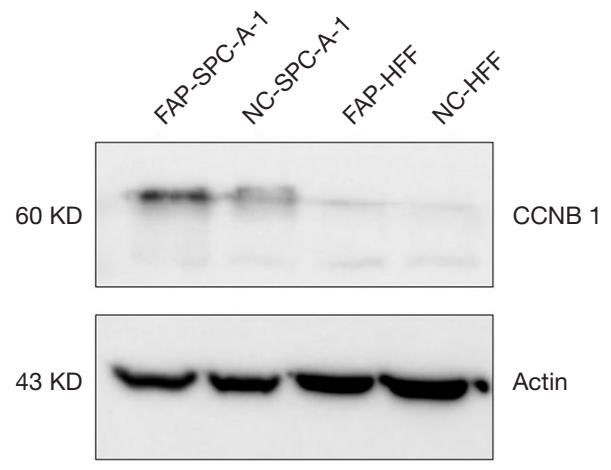

Figure 3 Effects of FAP- $\alpha$ on cell cycle, angiogenesis, and cell proliferation pathways. (A) PCR results showed that FAP- $\alpha$ could downregulate the expression of MMP3 in the HFF cell line; (B) PCR results showed that FAP- $\alpha$ could upregulate the expression of CCNB1 and CCNB2, while downregulate the expression of TGFA and EGF; (C) FAP- $\alpha$ could upregulate the expression of CCNB1 in the lung adenocarcinoma cell line (SPC-A-1). *, $\mathrm{P}<0.05$; **, $\mathrm{P}<0.01 ;{ }^{* * * *}, \mathrm{P}<0.0001$. in tumor stroma, the positive rate of low FAP- $\alpha$ expression was $46.74 \%$ ( 43 cases) with $34.9 \%$ of the number of events, while the positive rate of high FAP- $\alpha$ expression was $53.26 \%$ (49 cases) with $18.4 \%$ of the number of events in the tumor stroma (Tables $S 4, S 5$ ). Statistical results revealed that patients with higher expression of FAP- $\alpha$ in the stroma of lung adenocarcinoma correlated with poor prognosis $(\mathrm{P}=0.019)$ (Figure $4 B)$. As for the staining of FAP- $\alpha$ in the cytoplasm of tumor cells, the positive rate of low FAP- $\alpha$ expression was $68.48 \%$ ( 63 cases) with $28.6 \%$ of the number of events, while the positive rate of high FAP- $\alpha$ expression was $31.53 \%$ ( 29 cases) with $20.7 \%$ of the number of events in the tumor cytoplasm (Tables S6,S7). Survival analysis showed that there were no statistically significant differences between the high and low expression of FAP- $\alpha$ in the cytoplasm of lung adenocarcinoma $(\mathrm{P}=0.4)$ (Figure $4 C$ ). TCGA data also showed that FAP- $\alpha$ expression within tumor tissues was higher both in cytoplasm and stroma compared with normal tissues $(\mathrm{P}<0.05)$ (Figure $4 D)$.

\section{Discussion}

In clinical practice, non-small cell lung cancer accounts for $85 \%$ of all lung cancer mortalities (19). Due to their unique biological characteristics, prognosis, and sensitivity to radiotherapy and chemotherapy, lung squamouscell carcinoma and adenocarcinoma, the two major pathological types of NSCLC, remain the primary focus of clinical research in lung cancer. More urgently, lung adenocarcinoma has shown a greater metastatic potential at an early stage accompanied by soaring incidence $(19,20)$. Stirred by these trends, new pathological classifications of lung adenocarcinoma and its effect on prognosis have been subsequently established $(21,22)$. The histological classification of lung cancer is mainly determined by morphological characteristics, while the heterogeneity, prognosis, and treatment response of the tumor microenvironment in lung cancer have remained mostly neglected by clinicians. Therefore, a more comprehensive and detailed genotyping of lung cancer is needed to guide clinical treatments (23).

A previous study revealed that cancer-associated fibroblasts have a pro-tumorigenic role at all stages of disease progression (24). FAP, as an important surface marker of cancer-associated fibroblasts (CAFs), plays a vital role in promoting the progression of tumors and acts as an immune suppressor in the tumor microenvironment. 


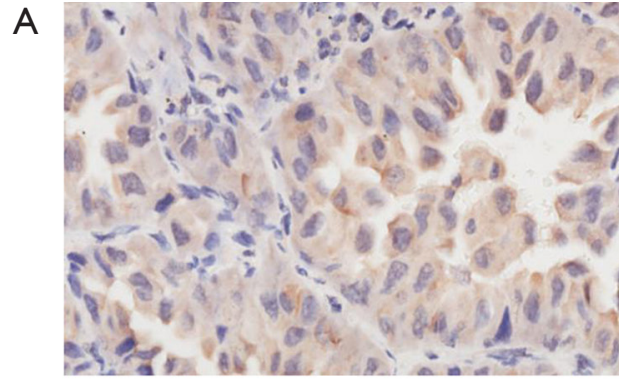

a

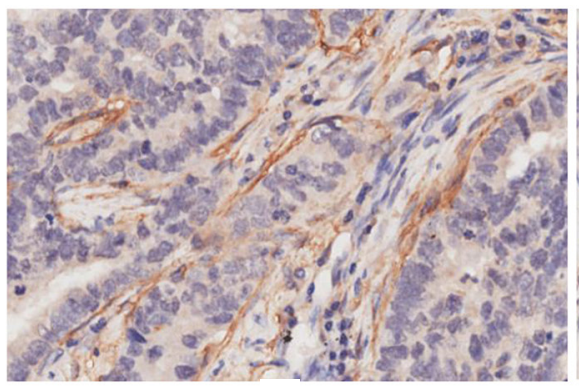

C

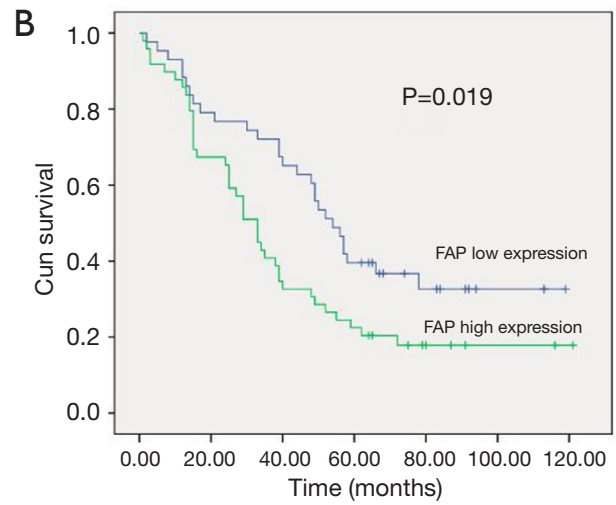

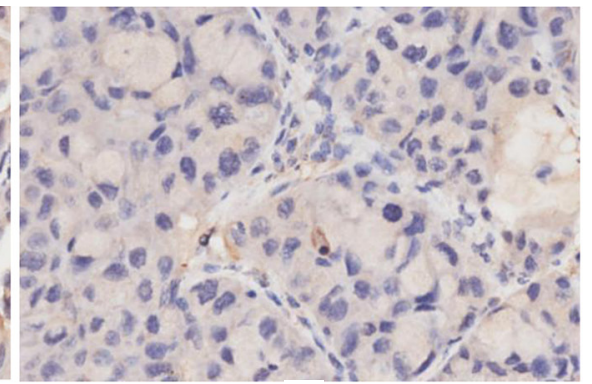

b

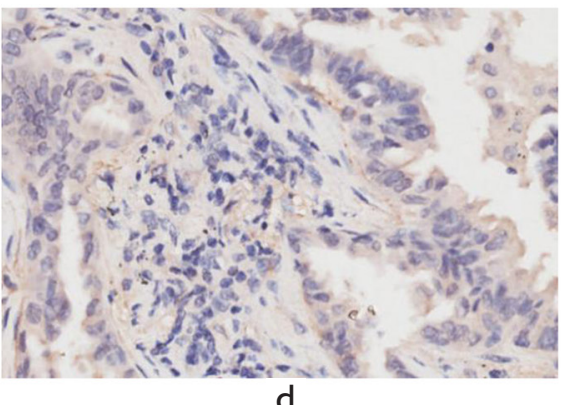

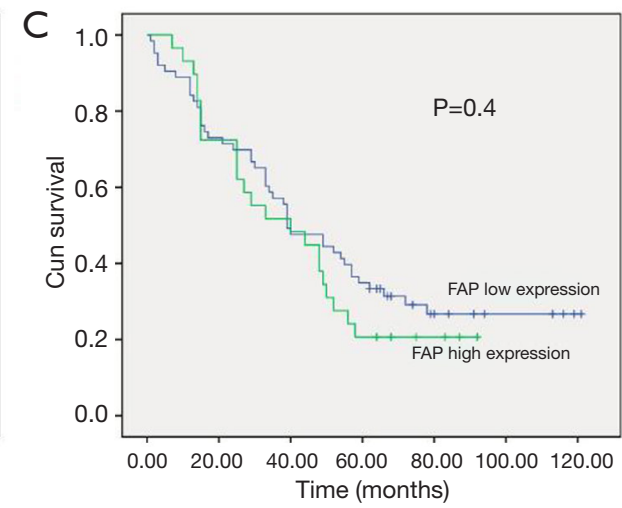

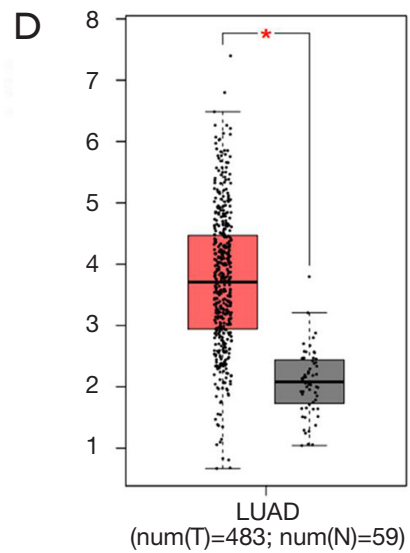

Figure 4 Expression and survival analysis of FAP- $\alpha$ in lung adenocarcinoma. (A) Fibroblast activation protein- $\alpha$ (FAP- $\alpha$ ) staining in lung adenocarcinoma tissues (20x), showing cytoplasmic staining (a, high expression of FAP- $\alpha$; b, low expression of FAP- $\alpha$ ) and stromal staining (c, high expression of FAP- $\alpha$; d, low expression of FAP- $\alpha$ ) of FAP- $\alpha$ in tumor tissue; (B) patients with a higher expression of FAP- $\alpha$ in tumor stroma had a poor prognosis $(\mathrm{P}=0.019)$; $(\mathrm{C})$ the prognosis of patients with different FAP- $\alpha$ expression in the tumor cytoplasm was not statistically significant $(\mathrm{P}=0.4)$; (D) TCGA data showed that FAP- $\alpha$ expression within tumor tissues was higher (in both cytoplasm and stroma) compared with normal tissues $(\mathrm{P}<0.05)$. * $\mathrm{P}<0.05$.

Table 1 Differential expression of FAP- $\alpha$ in tumor samples

\begin{tabular}{llc}
\hline Expression of FAP- $\alpha$ & Mean \pm SD & $P$ value \\
\hline Tumor stroma $(n=92)$ & $2.147 \pm 1.060$ & 0 \\
Stoma of adjacent tissue $(n=84)$ & $0.506 \pm 0.762$ & -7.443 \\
Tumor cytoplasm $(n=92)$ & $2.457 \pm 3.058$ & 0 \\
Cytoplasm of adjacent tissue $(n=83)$ & $0.012 \pm 0.077$ & -6.661 \\
\hline
\end{tabular}


Given these observations, it may thus be a promising target for cancer therapy $(15,25,26)$. However, the view of the pro-tumorigenic role of CAFs in cancer development has been challenged, and CAFs may in fact restrain cancer progression, implying a paradoxical interaction within the tumor microenvironment $(15,27)$. In our study, 75 patients with lung adenocarcinoma were recruited. Immunohistochemical analysis of FAP in the postoperative pathological specimens was performed with 5-10 years of follow-up. Statistical analyses revealed that the clinical data were well distributed, the age of patients was negatively correlated with FAP expressions $(\mathrm{P}=0.011)$, and the expression of FAP- $\alpha$ within tumor tissues was higher both in the cytoplasm and stroma compared with normal tissues $(\mathrm{P}<0.05)$.

Notably, our study showed that both cytoplasmic and interstitial staining of FAP could be found in lung adenocarcinoma tissues when compared with adjacent normal tissues. These findings suggest that FAP could be expressed both in stroma cells and lung adenocarcinoma cells, which is consistent with some previous research regarding the expression of FAP in some tumor cells including breast cancer cell lines (MDA-MB-435, MDAMB-436) (28), pancreatic cancer cell lines (SW1990, Miapaca-1,AsPC-1,BxPC-3) (29), and oral squamous cell carcinoma cell lines (KB, Tca-8113) (30). Survival analysis showed that patients with a higher expression of FAP in tumor stroma had a poor prognosis $(\mathrm{P}=0.019)$, while this situation was quite different in patients with a high expression of FAP in tumor cytoplasm $(\mathrm{P}=0.4)$. Presumably, the prognostic value of FAP in human adenocarcinoma was mainly determined by its main function in stroma cells, while FAP could also serve as an oncogene in tumor cells, which may facilitate tumor cells' proliferative ability.

Recent research of 59 cases reported the prognostic significance of FAP expression in non-small cell lung cancer (NSCLC) (including 27 cases of lung adenocarcinoma). The immunohistochemical analysis found the interstitial positive expression of FAP was $76 \%$, and survival analysis suggested that the expression of FAP was associated with adverse prognosis in patients $(\mathrm{P}<0.05)$. Meanwhile, FAP expression and the low differentiation of tumors were independent prognostic factors in multivariate analysis $(\mathrm{P}<0.05)$, but not for the histological types $(\mathrm{P}>0.05)$ (18). Unfortunately, there were only 3 years of follow-up and a small number of cases in this study. So, more in-depth, large-scale, long-term follow-up research is needed in the future.

FAP was first detected by Rettig and others in 1986 using monoclonal antibodies (F19) on the surface of activated fibroblasts. Human FAP contains two subunits: $\alpha$ subunit and $\beta$ subunit. Some components in the $\alpha$ subunit [serine (S624), aspartic acid (D702), and histidine (H734) are catalytic triplets] play a vital role in the biological activity of FAP, and the activity of $\alpha$-subunit mainly depends on its dimer structure (31). The specific markers of CAFs are still controversial. For example, FSP1, $\alpha$-SMA, FAP, PDGFR, and vimentin can be expressed on the surface of activated fibroblasts. It must be mentioned that the specificities of the aforementioned markers are not absolute. CAFs can be highly heterogeneous, with distinct expression patterns depending on different contexts (tumor sources, tumor staging, and so forth), and CAFs may have a combination of multiple markers at the same time (24).

Since the discovery of FAP, early research focused mainly on its structure, distribution, and double enzymatic activity. However, recent studies have shown that FAP could be over-expressed on the surface of certain tumor cells $(14,28-30)$. Some studies found that the proliferation and migration capacity of tumor cells increased significantly after the lentiviral transfection of the FAP-overexpression plasmid, suggesting that FAP could serve as an oncogene and activate potential signaling pathways like PI3K and FAK $(15,32,33)$, but results contradicting these findings have also been produced (15). Our study confirmed that the proliferation rates of human fibroblast strain FAP-HFF and FAP-BJ, and human lung adenocarcinoma cell line FAP-SPC-A-1 were higher than those of controls $(\mathrm{P}<0.05)$, indicating that FAP could promote the proliferation of both tumor cells and stromal cells. The underlying mechanism in SPC-A-1 cell line might be the increase of cell cycle protein CCNB1, which is a regulatory subunit of cell cycle-dependent kinase (CDK1) and can change the cell cycle from the G2 to M stage in normal cells. However, its regulatory effect is completely lost in tumor cells, and the proliferation of tumor cells is enhanced after its overexpression (34). CCNB1 now has been confirmed to be associated with tumor proliferation and prognosis in hepatocellular carcinoma and pancreatic cancer $(35,36)$. The proliferation of fibroblasts was also enhanced after the overexpression of FAP, which may be responsible for facilitating tumorigenesis and metastasis by remolding the extracellular matrix (33). Notably, after giving anti-fibrosis drugs (nintedanib) to patients with lung adenocarcinoma, the proliferation of tumor cells was attenuated, suggesting that CAFs in the microenvironment of lung cancer can promote tumorigenesis (37). 


\section{Conclusions}

Our study indicates that FAP- $\alpha$ can facilitate the proliferation of lung adenocarcinoma cells and might be a prognostic marker in human lung adenocarcinoma.

\section{Acknowledgments}

Funding: This study was supported by grants from the National Natural Science Foundation of China (No. 81360245, 81660477, and 81460436), the Yunnan Province Science and Technology Department (No. 2017 FE468 (007) and 2017FB149), and the Health Commission of Kunming City (2016SWJG).

\section{Footnote}

Conflicts of Interest: The authors have no conflicts of interest to declare.

Ethical Statement: The authors are accountable for all aspects of the work in ensuring that questions related to the accuracy or integrity of any part of the work are appropriately investigated and resolved. Animal care and experiments were performed in accordance with the guidelines of the Institutional Animal Committee of Kunming Medical University, and the procedure was approved by the Institutional Animal Care and Use Committee.

Open Access Statement: This is an Open Access article distributed in accordance with the Creative Commons Attribution-NonCommercial-NoDerivs 4.0 International License (CC BY-NC-ND 4.0), which permits the noncommercial replication and distribution of the article with the strict proviso that no changes or edits are made and the original work is properly cited (including links to both the formal publication through the relevant DOI and the license). See: https://creativecommons.org/licenses/by-nc-nd/4.0/.

\section{References}

1. Siegel RL, Miller KD, Jemal A. Cancer Statistics, 2017. CA Cancer J Clin 2017;67:7-30.

2. Hui L, Chen Y. Tumor microenvironment: Sanctuary of the devil. Cancer Lett 2015;368:7-13.

3. Verrecchia F, Redini F. Transforming Growth Factor-beta Signaling Plays a Pivotal Role in the Interplay Between
Osteosarcoma Cells and Their Microenvironment. Front Oncol 2018;8:133.

4. Sternlicht MD, Lochter A, Sympson CJ, et al. The stromal proteinase MMP3/stromelysin-1 promotes mammary carcinogenesis. Cell 1999;98:137-46.

5. Shue YT, Lim JS, Sage J. Tumor heterogeneity in small cell lung cancer defined and investigated in pre-clinical mouse models. Transl Lung Cancer Res 2018;7:21-31.

6. Choi H, Moon A. Crosstalk between cancer cells and endothelial cells: implications for tumor progression and intervention. Arch Pharm Res 2018;41:711-24.

7. Alsaab HO, Sau S, Alzhrani R, et al. PD-1 and PDL1 Checkpoint Signaling Inhibition for Cancer Immunotherapy: Mechanism, Combinations, and Clinical Outcome. Front Pharmacol 2017;8:561.

8. Zi F, He J, He D, et al. Fibroblast activation protein alpha in tumor microenvironment: recent progression and implications (review). Mol Med Rep 2015;11:3203-11.

9. Chung KM, Hsu SC, Chu YR, et al. Fibroblast activation protein (FAP) is essential for the migration of bone marrow mesenchymal stem cells through RhoA activation. PLoS One 2014;9:e88772.

10. Park JE, Lenter MC, Zimmermann RN, et al. Fibroblast activation protein, a dual specificity serine protease expressed in reactive human tumor stromal fibroblasts. J Biol Chem 1999;274:36505-12.

11. Wikberg ML, Edin S, Lundberg IV, et al. High intratumoral expression of fibroblast activation protein (FAP) in colon cancer is associated with poorer patient prognosis. Tumour Biol 2013;34:1013-20.

12. Ha SY, Yeo SY, Xuan YH, et al. The prognostic significance of cancer-associated fibroblasts in esophageal squamous cell carcinoma. PLoS One 2014;9:e99955.

13. Chen L, Chen M, Han Z, et al. Clinical significance of FAP-alpha on microvessel and lymphatic vessel density in lung squamous cell carcinoma. J Clin Pathol 2018;71:721-8.

14. Zeng C, Wen M, Liu X. Fibroblast activation protein in osteosarcoma cells promotes angiogenesis via AKT and ERK signaling pathways. Oncol Lett 2018;15:6029-35.

15. Wen Z, Liu Q, Wu J, et al. Fibroblast activation protein $\alpha$-positive pancreatic stellate cells promote the migration and invasion of pancreatic cancer by CXCL1-mediated Akt phosphorylation. Ann Transl Med 2019;7:532.

16. Wen X, He X, Jiao F, et al. Fibroblast Activation Proteinalpha-Positive Fibroblasts Promote Gastric Cancer Progression and Resistance to Immune Checkpoint Blockade. Oncol Res 2017;25:629-40. 
17. Huang Q, Li J, Wei A. Identification of potential therapeutic targets in hepatocellular carcinoma using an integrated bioinformatics approach. Transl Cancer Res 2018;7:849-58.

18. Liao Y, Ni Y, He R, et al. Clinical implications of fibroblast activation protein-alpha in non-small cell lung cancer after curative resection: a new predictor for prognosis. J Cancer Res Clin Oncol 2013;139:1523-8.

19. Tsuboi M, Ohira T, Saji H, et al. The present status of postoperative adjuvant chemotherapy for completely resected non-small cell lung cancer. Ann Thorac Cardiovasc Surg 2007;13:73-7.

20. Cross MD. Advances in NSCLc: histologic distinction between adenocarcinoma and squamous cell carcinoma. MLO Med Lab Obs 2012;44:40-2, 44.

21. Travis WD, Brambilla E, Noguchi M, et al. International Association for the Study of Lung Cancer/American Thoracic Society/European Respiratory Society: international multidisciplinary classification of lung adenocarcinoma: executive summary. Proc Am Thorac Soc 2011;8:381-5.

22. Miyoshi T, Satoh Y, Okumura S, et al. Early-stage lung adenocarcinomas with a micropapillary pattern, a distinct pathologic marker for a significantly poor prognosis. Am J Surg Pathol 2003;27:101-9.

23. Luo X, Zang X, Yang L, et al. Comprehensive Computational Pathological Image Analysis Predicts Lung Cancer Prognosis. J Thorac Oncol 2017;12:501-9.

24. Kalluri R. The biology and function of fibroblasts in cancer. Nat Rev Cancer 2016;16:582-98.

25. Zhang Y, Ertl HC. Depletion of FAP+ cells reduces immunosuppressive cells and improves metabolism and functions CD8+T cells within tumors. Oncotarget 2016;7:23282-99.

26. Yang X, Lin Y, Shi Y, et al. FAP Promotes Immunosuppression by Cancer-Associated Fibroblasts in the Tumor Microenvironment via STAT3-CCL2 Signaling. Cancer Res 2016;76:4124-35.

27. Ramirez-Montagut T, Blachere NE, Sviderskaya EV, et al. FAPalpha, a surface peptidase expressed during wound

Cite this article as: Shi J, Hou Z, Yan J, Qiu W, Liang L, Meng M, Li L, Wang X, Xie Y, Jiang L, Wang W. The prognostic significance of fibroblast activation protein- $\alpha$ in human lung adenocarcinoma. Ann Transl Med 2020;8(5):224. doi: $10.21037 /$ atm.2020.01.82 healing, is a tumor suppressor. Oncogene 2004;23:5435-46.

28. Goodman JD, Rozypal TL, Kelly T. Seprase, a membranebound protease, alleviates the serum growth requirement of human breast cancer cells. Clin Exp Metastasis 2003;20:459-70.

29. Shi M, Yu DH, Chen Y, et al. Expression of fibroblast activation protein in human pancreatic adenocarcinoma and its clinicopathological significance. World J Gastroenterol 2012;18:840-6.

30. Wang H, Wu Q, Liu Z, et al. Downregulation of FAP suppresses cell proliferation and metastasis through PTEN/PI3K/AKT and Ras-ERK signaling in oral squamous cell carcinoma. Cell Death Dis 2014;5:e1155.

31. Wonganu B, Berger BW. A specific, transmembrane interface regulates fibroblast activation protein (FAP) homodimerization, trafficking and exopeptidase activity. Biochim Biophys Acta 2016;1858:1876-82.

32. Jia J, Martin TA, Ye L, et al. FAP-alpha (Fibroblast activation protein-alpha) is involved in the control of human breast cancer cell line growth and motility via the FAK pathway. BMC Cell Biol 2014;15:16.

33. Lee HO, Mullins SR, Franco-Barraza J, et al. FAPoverexpressing fibroblasts produce an extracellular matrix that enhances invasive velocity and directionality of pancreatic cancer cells. BMC Cancer 2011;11:245.

34. Yuan J, Kramer A, Matthess Y, et al. Stable gene silencing of cyclin B1 in tumor cells increases susceptibility to taxol and leads to growth arrest in vivo. Oncogene 2006;25:1753-62.

35. Wu J, Zhou X, Fan Y, et al. Long non-coding RNA 00312 downregulates cyclin B1 and inhibits hepatocellular carcinoma cell proliferation in vitro and in vivo. Biochem Biophys Res Commun 2018;497:173-80.

36. Zhou L, Li J, Zhao YP, et al. The prognostic value of Cyclin B1 in pancreatic cancer. Med Oncol 2014;31:107.

37. Gabasa M, Ikemori R, Hilberg F, et al. Nintedanib selectively inhibits the activation and tumour-promoting effects of fibroblasts from lung adenocarcinoma patients. Br J Cancer 2017;117:1128-38. 
A

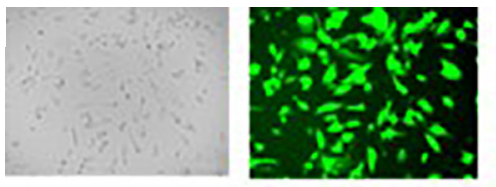

FAP-HFF (100x)

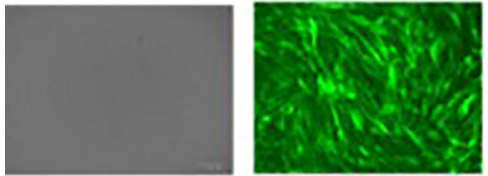

NC-HFF (100x)

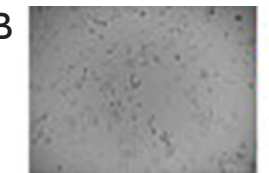

FAP-BJ $(100 x)$

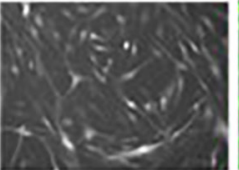

NC-BJ (100x)
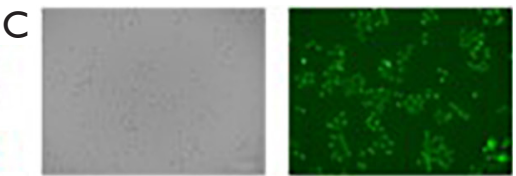

FAP-SPC-A-1 (100x)

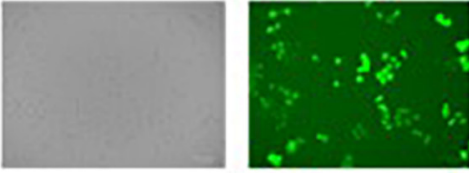

NC-SPC-A-1 (100x)

Figure S1 (A,B,C) After transfected with FAP-overexpressing and negative plasmids, the expression of GFP in HFF, BJ, and SPC-A-1 cells was observed under a fluorescence microscope (100x). The transfection efficiency was more than $80 \%$, and the cell status was normal.

Table S1 Survival analysis of clinicopathological variables in human samples (Kaplan-Meier and log-rank analysis)

\begin{tabular}{|c|c|c|c|c|}
\hline Variables & Total No. & No. of Events & Chi-Square & $P$ value \\
\hline Age (years) & & & 0.0001 & 0.995 \\
\hline$\leq 65$ & $61(64.8)$ & 45 (64.29) & & \\
\hline$>65$ & $33(35.2)$ & $25(35.71)$ & & \\
\hline Gender & & & 0.897 & 0.344 \\
\hline Male & $51(54.3)$ & $39(55.71)$ & & \\
\hline Female & $43(45.7)$ & $31(44.29)$ & & \\
\hline Pathological grade & & & 1.551 & 0.460 \\
\hline I & $3(3.19)$ & $1(1.43)$ & & \\
\hline II & 62 (65.96) & 48 (68.57) & & \\
\hline III & $29(30.85)$ & $21(30.0)$ & & \\
\hline Tumor size (cm) & & & 5.733 & 0.017 \\
\hline$\leq 5$ & $76(80.85)$ & $55(78.57)$ & & \\
\hline$>5$ & $18(19.15)$ & $15(21.43)$ & & \\
\hline T stage & & & 11.842 & 0.008 \\
\hline $\mathrm{T} 1$ & $19(20.21)$ & $10(14.29)$ & & \\
\hline T2 & 52 (55.32) & $41(58.57)$ & & \\
\hline T3 & $17(18.09)$ & $13(18.57)$ & & \\
\hline $\mathrm{T} 4$ & $6(6.38)$ & $6(8.57)$ & & \\
\hline N stage & & & 23.144 & 0.0001 \\
\hline No & 39 (51.32) & $22(40.0)$ & & \\
\hline $\mathrm{N} 1$ & $16(21.05)$ & $13(23.64)$ & & \\
\hline N2 & $16(21.05)$ & $15(27.27)$ & & \\
\hline N3 & $5(6.58)$ & $5(9.09)$ & & \\
\hline M stage & & & 0.019 & 0.891 \\
\hline Mo & 92 (98.92) & 68 (98.55) & & \\
\hline M1 & $1(1.08)$ & $1(1.45)$ & & \\
\hline TNM stage & & & 22.174 & 0.0001 \\
\hline TNMI & $28(31.46)$ & 15 (22.39) & & \\
\hline TNMII & $30(33.71)$ & $23(34.33)$ & & \\
\hline TNMIII & $30(33.71)$ & $28(41.79)$ & & \\
\hline TNMIV & $1(1.12)$ & $1(1.49)$ & & \\
\hline
\end{tabular}


Table S2 Correlation between clinical data and expression of FAP- $\alpha$ in tumor stroma (Spearman's and Pearson correlation)

\begin{tabular}{lccc}
\hline Variable & Correlation coefficient & Number & P value \\
\hline Sex & -0.11 & 92 & 0.297 \\
Age & -0.266 & 92 & 0.011 \\
Tumor size & 0.148 & 92 & 0.158 \\
Grade & 0.039 & 92 & 0.948 \\
T stage & 0.108 & 92 & 0.307 \\
N stage & 0.050 & 74 & 0.67 \\
M stage & -0.020 & 91 & 0.853 \\
TNM stage & 0.165 & 87 & 0.126 \\
\hline
\end{tabular}

The clinicopathological analysis revealed that the interstitial expression of FAP- $\alpha$ was only negatively correlated with the age of patients $(P=0.011)$. FAP, fibroblast activation protein.

Table S3 Correlation between clinical data and the expression of FAP- $\alpha$ in tumor cytoplasma (Spearman's and Pearson correlation)

\begin{tabular}{lccc}
\hline Variable & Correlation coefficient & Number & P value \\
\hline Sex & 0.007 & 92 & 0.946 \\
Age & -0.056 & 92 & 0.595 \\
Tumor size & -0.114 & 92 & 0.281 \\
Grade & 0.113 & 92 & 0.283 \\
T stage & 0.103 & 74 & 0.327 \\
N stage & 0.034 & 91 & 0.772 \\
M stage & 0.041 & 87 & 0.700 \\
TNM stage & 0.023 & 0.836
\end{tabular}

The clinicopathological analysis revealed that there was no correlation between clinical data and FAP- $\alpha$ expression in tumor cytoplasma.

FAP, fibroblast activation protein.

Table S4 Expression of FAP- $\alpha$ in the stroma of lung adenocarcinoma

\begin{tabular}{lcccc}
\hline \multirow{2}{*}{ FAP- $\alpha$ expression } & Total No. & No. of events & \multicolumn{2}{c}{ Censored } \\
\cline { 3 - 5 } & & 43 & 28 & N \\
Low expression & 49 & 40 & 15 & 34.9 \\
High expression & 92 & 68 & 9 & 24 \\
Total & 92 & 26.1 \\
\hline
\end{tabular}

FAP, fibroblast activation protein.

Table S5 Overall comparisons

\begin{tabular}{lccc}
\hline & Chi-Square & df & P value \\
\hline Log rank (Mantel-Cox) & 5.486 & 1 & 0.019 \\
\hline
\end{tabular}


Table S6 Expression of FAP- $\alpha$ in the cytoplasm of lung adenocarcinoma cells

\begin{tabular}{lcccc}
\hline & Total No. & No. of events & \multicolumn{2}{c}{ Censored } \\
\cline { 3 - 5 } FAP- $\alpha$ expression & & 45 & No. & 18 \\
\hline Low expression & 63 & 23 & 6 & 28.6 \\
High expression & 29 & 68 & 24 & 26.1 \\
Total & 92 & & 24 \\
\hline
\end{tabular}

FAP, fibroblast activation protein.

Table S7 Overall comparisons

\begin{tabular}{lccc}
\hline & Chi-Square & df & P value \\
\hline Log rank (Mantel-Cox) & 0.709 & 1 & 0.4 \\
\hline
\end{tabular}

